\section{CASE REPORT}

\author{
E.N. Mussak
}

A.I. Holodny

S.K. Karimi

\title{
Meningioma of the Mandible: Imaging with CT
}

SUMMARY: We present the first description of $\mathrm{CT}$ of a meningioma of the mandible in the literature. Extracranial meningiomas are exceedingly rare. There have been 3 cases of meningioma of the mandible described in the literature, but none characterized with cross-sectional imaging. We describe the clinical and CT features used to establish the diagnosis as well as how to differentiate this lesion from other pathology of the mandible.

0 nly 3 cases of a meningioma of the mandible have been described in the literature. ${ }^{1,2}$ The mandibular meningiomas are classified as primary extracranial meningiomas. Extracranial meningiomas are usually an extension of an intracranial tumor but can arise independently, such as meningioma of the mandible. The independent formation of these tumors is believed to involve meningocytes along the sheaths of cranial nerves as they exit the skull foramina, fibroblastic mesenchymal cell transformation, or displaced arachnoid cap cells. Metastasis from a central nervous system (CNS) meningioma can naturally give rise to an "ectopic" meningioma as well. Extracranial meningiomas are exceptionally rare. To our knowledge, we report the first CT findings of a mandibular meningioma.

\section{Case Report}

A 62-year-old man presented with fullness on the left mid-body of the mandible. The patient's history dated to 2 years earlier when he sought consultation from his dentist. A swelling was noted on his left lower gum with mild pain, which was clinically thought to represent an abscess. Plain films revealed a large well-delineated radiolucent lesion involving the mandibular body. He was treated with 4 different antibiotic regimens during the following year with no regression of the mass. The patient denied any bleeding from the oral cavity or any problems with speech or mastication. At that point, his primary care physician referred him to an otolaryngologist for further management and work-up of the mass.

The work-up included a CT of the mandible without contrast, which showed a well-circumscribed lytic expansile mass involving the mandibular body on the left side, which broke through the cortex at several different sites. The mass measured approximately $7 \times 3 \mathrm{~cm}$ (anteroposterior $\times$ transverse) and spanned from the alveolar ridge to the inferior cortex. Posteriorly, the lesion extended $1 \mathrm{~cm}$ from the angle of the mandible and anteriorly $1.8 \mathrm{~cm}$ from the mentum (Fig 1). There was no evidence of gross tumor extending into the infratemporal fossa along the course of $\mathrm{V} 3$, and the foramen ovale was normal on the CT scan.

A fine-needle aspiration of the mass was performed. Cytologic analysis revealed neoplastic spindle cells present with minimal nuclear and cytologic atypia. The sample was reviewed at an intradepart-

\section{Received August 16, 2006; accepted September 20.}

From Weill Medical College of Cornell University (E.N.M.), New York; and the Department of Radiology (A.I.H., S.K.), Neuroradiology Service, Memorial Sloan Kettering Cancer Center, NY.

Please address correspondence to Sasan Karimi, MD, Department of Radiology, Memorial Sloan-Kettering Cancer Center, 1275 York Ave, New York, NY 10021; e-mail: karimis@mskcc.org

DOI 10.3174/ajnr.A0503

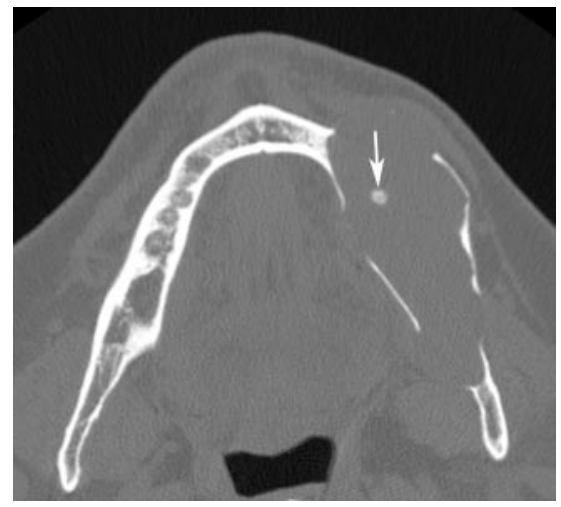

Fig 1. Axial CT image in bone window parallel to the long axis of the body of the mandible. The expansile meningioma erodes through the cortex and contains focal calcification (arrow)

mental pathology conference and was determined to represent an extracranial meningioma. The patient was then recommended for segmental mandibulectomy and mandibular reconstruction with a fibula free flap.

Postoperatively, the left submandibular gland and left mandible were submitted to surgical pathology for analysis. The salivary gland was found to be benign, but the mandible showed a low-grade spindle cell neoplasm consistent with meningioma (Fig 2). It was unencapsulated and was present within the mandibular bone extending through the cortex into the gingival submucosa as well as abutting surrounding skeletal muscle. Mitoses were not readily identifiable, and the possibility of malignancy was unlikely. There was no tumor necrosis, and all margins of resection were negative. Furthermore, on pathology, there was no perineural invasion and no evidence of involvement of the inferior alveolar nerve.

\section{Discussion}

Meningiomas usually originate from meningothelial cells of the arachnoid layer that constitute the external lining of the brain and arise mostly at the base of the skull, in the parasellar regions as well as along the cerebral convexities. In general, these lesions are solitary with the exception of individuals with neurofibromatosis, for whom multiple lesions are common. Intracranial meningiomas come to clinical attention as a result of symptoms, including hemiparesis, seizure, or cranial neuropathy such as vision loss. These symptoms are the result of compression of surrounding neural tissues by the meningioma. Extracranial meningiomas present most frequently in the orbit, paranasal sinuses, temporal bone, and oral cavity in that order. ${ }^{3}$ The most common presentation of all extracranial meningiomas is a change in cutaneous expansion or color.

Both intracranial and extracranial meningiomas may be 


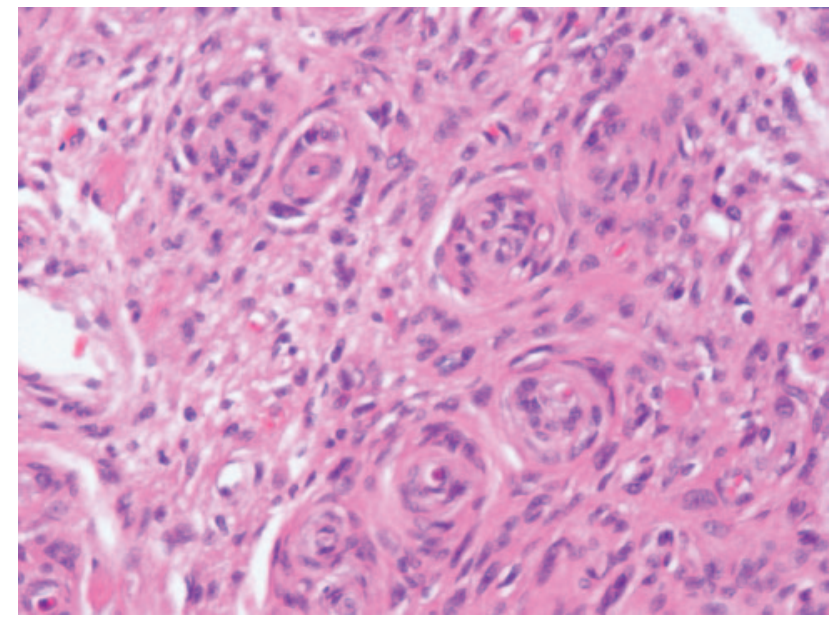

Fig 2. Photomicrograph demonstrates a cellular neoplasm, comprising spindled cells with indistinct cytoplasmic borders and minimal nuclear pleomorphism arranged in whorls, characteristic of meningioma (hematoxylin-eosin, original magnification $\times 200$ ).

categorized as benign (90\%), atypical/borderline (5\%), and malignant $(5 \%)$. In the benign category, there are several histopathologic subtypes, including syncytial, fibrous, and transitional. The current case and the previously described cases of mandibular meningiomas were unencapsulated and consisted of elongated spindle cells that make up larger whorl-like collections. No mitotic figures have been noted in the small number of cases of mandibular meningioma. ${ }^{1,2}$

Extracranial meningiomas are most often due to extension from an intracranial primary lesion and occur much more infrequently in an independent fashion. These independent meningiomas with no association with intracranial tumors can arise in 4 ways. They may arise from meningocytes along the sheaths of cranial nerves when they exit the skull foramina, from fibroblastic mesenchymal cell transformation, displaced embryonic rests of arachnoid cells, or from a metastasis from a primary CNS meningioma. ${ }^{4}$

Metastasis from a CNS meningioma is the least likely reason for an extracranial meningioma in the head and neck region. ${ }^{5}$ Because there was no continuity with the intracranial region and no primary brain lesion present on CT, the mandibular lesion probably arose from transformed fibroblasts or displaced arachnoid cells. Lang et $\mathrm{al}^{6}$ proposed a single term "primary extradural meningioma" for these lesions. Primary extradural meningiomas have been reported to occur in the calvaria, ${ }^{7}$ scalp, ${ }^{8}$ nasopharynx,${ }^{9}$ orbit, ${ }^{10}$ paranasal sinuses, ${ }^{11}$ neck, ${ }^{12}$ and skin. ${ }^{13}$ Rare meningiomas occurring in the lung, ${ }^{14}$ mediastinum, ${ }^{15}$ phalanges, ${ }^{16}$ and paraspinal region ${ }^{17}$ have also been reported.

The first case of meningioma of the mandible was reported in 1992 by Landini and Kitano. ${ }^{1}$ They proposed that with the mandibular nerve being prone to other types of neural tumors, it may be in some way predisposed to the development of primary extradural meningiomas. For instance, one third of intraosseous neurilemomas and neurofibromas occur in the mandible possibly due to the length and size of the mandibular nerve. $^{18}$

The CT images of the present case showed multiple regions of cortical breakthrough and scalloping of the residual cortex. A small focus of calcification was also present within the le-

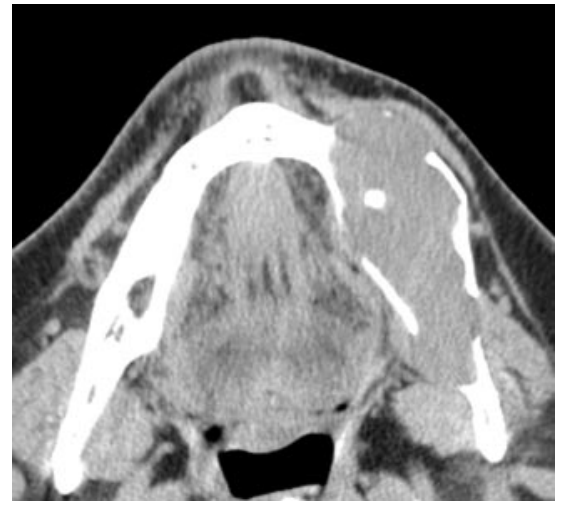

Fig 3. Axial CT image in soft-tissue window at the same level as Fig 1 demonstrates essentially homogeneous internal soft-tissue attenuation without any fluid-fluid levels.

sion, which did not appear to represent a tooth or an eroded tooth (Fig 1). There were no associated unerupted teeth. Otherwise, the internal density of the lesion was homogeneous and slightly less attenuated than muscle, without any fluidfluid levels (Fig 3). Overall the mass had a nonaggressive appearance on the CT scan. Internal calcification is a common finding in meningiomas; however, because of the rarity of meningioma involving the mandible, the internal calcification may not be a good indicator of the radiographic diagnosis.

The CT appearance is also suggestive of an ameloblastoma because ameloblastomas are low attenuation without matrix mineralization. The lesion was not associated with an unerupted tooth, suggesting a dentigerous cyst, and did not contain fluid-fluid levels as one would expect with an aneurysmal bone cyst and giant cell tumor. An unusually large odontogenic keratocyst or dentigerous cyst can resemble the meningioma in this case, but dentigerous and odontogenic keratocysts tend to demonstrate lower internal densities. In a case of an abscess/osteomyelitis, one would expect mixed lytic and sclerotic bony changes of osteomyelitis and perhaps a sequestrum as well as associated inflammatory soft-tissue changes, which were not present in this case. On the basis of the age of the patient, plasmacytoma and metastasis should be included in the differential diagnosis. Due to the orientation of the lesion along the long axis of the mandible, one might also consider a lesion arising from the inferior alveolar nerve such as a neurofibroma or perineurioma. It is important to keep in mind that the differential diagnosis of primary lesions of the mandible is broad. ${ }^{19}$ This is due to the fact that lesions can arise from the bone, nerves, vasculature, or dental elements.

The treatment of choice for mandibular meningioma involves surgical excision with good results with this patient and in the 3 previous cases. The current patient and 1 previously reported case were treated with resection preceding bone graft reconstruction. ${ }^{1}$ Excisional biopsy has been effective in 2 previous cases. ${ }^{2}$ Although the number of cases of meningioma of the mandible has been small, all tumors have been nonaggressive. Resectioning with reconstruction and excisional biopsy have proved to be well tolerated by the patient with no recurrence.

\section{References}

1. Landini G, Kitano M. Meningioma of the mandible. Cancer 1992;69:2917-20 2. Jones AC, Freedman PD. Primary extracranial meningioma of the mandible: a 
report of 2 cases. Oral Surg Oral Med Oral Pathol Oral Radiol Endod 2001;91:338-41

3. O'Reilly RC, Kapadia SB, Kamerer DB. Primary extracranial meningioma of the temporal bone. Otolaryngol Head Neck Surg 1998;118:690-94

4. Shuangshoti S, Netsky MG, Fitz-Hugh GS. Parapharyngeal meningioma with special reference to cell of origin. Ann Otol Rhinol Laryngol 1971;80:464-73

5. Shuangshoti S, Hongsaprabhas C, Netsky MG. Metastasizing meningioma. Cancer 1970;26:832-41

6. Lang FF, Macdonald OK, Fuller GN, et al. Primary extradural meningiomas: a report on nine cases and review of literature from the era of computerized tomography scanning. J Neurosurg 2000;93:940-50

7. Siegel GJ, Anderson PJ. Extracalvarial meningioma: case report. J Neurosurg $1966 ; 25: 83-86$

8. Lopez DA, Silvers DN, Helwig EB. Cutaneous meningiomas: a clinicopathologic study. Cancer 1974;34:728-44

9. Mori S, Kobayashi S, Miki H. Extracranial meningioma in the parapharyngeal space. Acta Pathol Jpn 1993;43:130-34

10. Karp LA, Zimmerman LE, Borit A. Primary intraorbital meningiomas. Arch Ophthalmol 1974;91:24-28
11. Thompson LD, Gyure KA. Extracranial sinonasal tract meningiomas: a clinicopathologic study of 30 cases with a review of the literature. Am J Surg Pathol 2000;24:640-50

12. Ibrahim AW, Satti MB, Ibrahim EM. Extraspinal meningioma: case report J Neurosurg 1986;64:328-30

13. Theaker JM, Fleming KA. Meningioma of the scalp: a case report with immunohistological features. J Cutan Pathol 1987;14:49-53

14. Strimlan CV, Golembiewski RS, Celko DA. Primary pulmonary meningioma Surg Neurol 1988;29:410-13

15. Wilson AJ, Ratliff JL, Lagios MD. Mediastinal meningioma. Am J Surg Pathol 1979;3:557-62

16. Daugaard S. Ectopic meningioma of a finger: case report. J Neurosurg 1983;58:778-80

17. Junaid TA, Nkposong EO, Kolawole TM. Cutaneous meningiomas and an ovarian fibroma in a three-year-old girl. J Pathol 1972;108:165-67

18. Eversole LR. Central benign and malignant neural neoplasms of the jaws: a review. J Oral Maxillofac Surg 1969;27:716-21

19. Kramer IRH, Pindborg JJ, Shear M. Histological Typing of Odontogenic Tumors 2nd ed. New York: Springer-Verlag; 1992:2-9 


\section{Erratum}

In the article published in the June-July 2007 issue titled "Meningioma of the Mandible: Imaging with CT" (Mussak EN, Holodny AI, Karimi S. AJNR Am J Neuroradiol 2007;28:1157-59), the authors deeply regret the inadvertent omission of Dr. Diane L. Carlson's name from the authors' list due to submission of incorrect files. Dr. Carlson's contribution to the manuscript, particularly in terms of pathologic discussion and imaging, was most significant and this work would have never been accomplished without her help.

DOI 10.3174/ajnr.A1341 